\title{
CHARACTERIZATION OF GENERALIZED ORLICZ SPACES
}

\author{
RITA FERREIRA, PETER HÄSTÖ, AND ANA MARGARIDA RIBEIRO
}

\begin{abstract}
The norm in classical Sobolev spaces can be expressed as a difference quotient. This expression can be used to generalize the space to the fractional smoothness case. Because the difference quotient is based on shifting the function, it cannot be used in generalized Orlicz spaces. In its place, we introduce a smoothed difference quotient and show that it can be used to characterize the generalized Orlicz-Sobolev space. Our results are new even in Orlicz spaces and variable exponent spaces.
\end{abstract}

\section{INTRODUCTION}

Bourgain, Brézis, and Mironescu [5, 6] studied the limit behavior of the Gagliardo semi-norms

$$
\|f\|_{W^{s, p}}^{p}=\int_{\Omega} \int_{\Omega} \frac{|f(x)-f(y)|^{p}}{|x-y|^{n+s p}} d x d y, \quad 0<s<1,
$$

as $s \rightarrow 1$, and established the appropriate scaling factor for comparing the limit with the $L^{p}$-norm of the gradient of $f$. They characterized the Sobolev space $W^{1, p}$ and proved the convergence of certain imaging models of Aubert and Kornprobst [2] to the well-known total variation model of Rudin, Osher, and Fatemi [31]. Our aim in this paper is to extend the characterization to generalized Orlicz spaces defined on open subsets of $\mathbb{R}^{n}$.

Generalized Orlicz spaces $L^{\varphi(\cdot)}$ have been studied since the 1940's. A major synthesis of functional analysis in these spaces is given in the monograph of Musielak [28] from 1983, for which reason they have also been called Musielak-Orlicz spaces. These spaces are similar to Orlicz spaces, but defined by a more general function $\varphi(x, t)$ that may vary with the location in space: the norm is defined by means of the integral

$$
\int_{\mathbb{R}^{n}} \varphi(x,|f(x)|) d x
$$

whereas in an Orlicz space, $\varphi$ would be independent of $x, \varphi(|f(x)|)$. When $\varphi(t)=t^{p}$, we obtain the Lebesgue spaces, $L^{p}$. Generalized Orlicz spaces are motivated by applications to image processing [7, 20], fluid dynamics [32], and differential equations [4, 17]. Recently, harmonic analysis in this setting has been studied e.g. in [12, 22, 26].

We have in mind two principal classes of examples of generalized Orlicz spaces: variable exponent spaces $L^{p(\cdot)}$, where $\varphi(x, t):=t^{p(x)}[11,14]$, and dual phase spaces, where $\varphi(x, t):=t^{p}+a(x) t^{q}[3,4,8,9,10]$. It is interesting to note that our general methods give optimal results in these two disparate cases, cf. [22]. Also covered are variants of the variable exponent case such as $t^{p(x)} \log (e+t)[17,27,29,30]$.

It is not difficult to see that a direct generalization of the difference quotient to the nontranslation invariant generalized Orlicz case is not possible [24, Section 1]. For instance Besov and Triebel-Lizorkin spaces in this context have been defined using Fourier theoretic approach [1, 15]. Hästö and Ribeiro [24], following [13], adopted a more direct approach with a smoothed difference quotient expressed by means of the sharp averaging operator

Date: December 15, 2016.

2010 Mathematics Subject Classification. 46E35, 46E30.

Key words and phrases. Musielak-Orlicz spaces, Orlicz space, Sobolev space, variable exponent, Poincaré inequality. 
$M_{B(x, r)}^{\#}$. This is the general approach adopted also in this paper. This paper improves [24] in three major ways:

1. Instead of variable exponent spaces, we consider more general generalized Orlicz spaces;

2. Instead of $\mathbb{R}^{n}$, we allow arbitrary open sets $\Omega \subset \mathbb{R}^{n}$; and

3. In our main result, we relax the technical assumption $f \in L^{1}(\Omega)$ to its natural form; i.e., $f \in L_{\mathrm{loc}}^{1}(\Omega)$.

The latter two generalizations have been previously established in the case $L^{p}$ by Leoni and Spector [25], see also [16, Section 1]. In order to achieve these goals, the methods of the main results (Section 4) are completely different from those in [24] and involve a new bootstrapping scheme.

We introduce some notation to state our main result. We refer to the next section for the precise definition of $L^{\varphi(\cdot)}$ and the assumptions in the theorem. Let $\Omega \subset \mathbb{R}^{n}$ be an open set, $\varphi \in \Phi_{w}(\Omega)$, and $\varepsilon>0$. Let $\psi_{\varepsilon}$ be a set of functions such that

$$
\psi_{\varepsilon} \in L^{1}(0, \infty), \quad \psi_{\varepsilon} \geqslant 0, \quad \int_{0}^{\infty} \psi_{\varepsilon}(r) d r=1
$$

and, for every $\gamma>0$,

$$
\lim _{\varepsilon \rightarrow 0^{+}} \int_{\gamma}^{\infty} \psi_{\varepsilon}(r) d r=0 .
$$

We define a weak quasi-semimodular, $\varrho_{\#, \Omega}^{\varepsilon}(f)$, on $L_{\text {loc }}^{1}(\Omega)$ by setting

$$
\varrho_{\#, \Omega}^{\varepsilon}(f):=\int_{0}^{\infty} \int_{\Omega_{r}} \varphi\left(x, \frac{1}{r} M_{B(x, r)}^{\#} f\right) d x \psi_{\varepsilon}(r) d r
$$

for $f \in L_{\text {loc }}^{1}(\Omega)$, where $\Omega_{r}:=\{x \in \Omega: \operatorname{dist}(x, \partial \Omega)>r\}$ and

$$
M_{B(x, r)}^{\#} f:=f_{B(x, r)}\left|f(y)-f_{B(x, r)}\right| d y \quad \text { with } f_{B(x, r)}:=f_{B(x, r)} f(y) d y .
$$

The associated quasi-norm, $\|f\|_{\#, \Omega}^{\varepsilon}$, is defined by

$$
\|f\|_{\#, \Omega}^{\varepsilon}:=\inf \left\{\lambda>0 \mid \varrho_{\#, \Omega}^{\varepsilon}(f / \lambda) \leqslant 1\right\} .
$$

The following is our main result stated for a $\Phi$-function - it is also possible to state it for weak $\Phi$-functions, see Theorem 4.6. The proof follows from Propositions 4.1 and 4.5.

Theorem 1.1. Let $\Omega \subset \mathbb{R}^{n}$ be an open set, let $\varphi \in \Phi(\Omega)$ satisfy Assumptions (A), (aInc), and $(\mathrm{aDec})$, and let $\left(\psi_{\varepsilon}\right)_{\varepsilon}$ be a family of functions satisfying (1.1) and (1.2). Assume that $f \in L_{\mathrm{loc}}^{1}(\Omega)$. Then,

$$
\nabla f \in L^{\varphi(\cdot)}\left(\Omega ; \mathbb{R}^{n}\right) \Leftrightarrow \limsup _{\varepsilon \rightarrow 0^{+}} \varrho_{\#, \Omega}^{\varepsilon}(f)<\infty .
$$

In this case,

$$
\lim _{\varepsilon \rightarrow 0^{+}} \varrho_{\#, \Omega}^{\varepsilon}(f)=\varrho_{\varphi(\cdot), \Omega}\left(c_{n}|\nabla f|\right) \quad \text { and } \quad \lim _{\varepsilon \rightarrow 0^{+}}\|f\|_{\#, \Omega}^{\varepsilon}=c_{n}\|\nabla f\|_{\varphi(\cdot), \Omega},
$$

where $c_{n}:=f_{B(0,1)}\left|x \cdot e_{1}\right| d x$.

Remark 1.2. Note that the previous result is new even in the case of classical Orlicz spaces. In this case, Assumption (A) automatically holds. 
Remark 1.3. In the case $\varphi(x, t)=t^{p(x)}$, Assumptions (A0) and (loc) always hold, while Assumptions (A1) and (A2) are equivalent to the local log-Hölder continuity and Nekvinda's decay condition, respectively. Moreover, if $p_{-}:=\inf _{x \in \Omega} p(x)>1$, then (aInc) holds; and if $p_{+}:=\sup _{x \in \Omega} p(x)<\infty$, then (aDec) holds.

\section{Preliminaries}

This section is organized as follows. In Subsection 2.1, we collect some notation used throughout this paper. Then, in Subsection 2.2, we recall the definition of $\Phi$-functions and of some of its generalizations; we recall also the associated Orlicz spaces, norms, and semimodulars. Finally, in Subsection 2.3, we introduce and discuss our main assumptions on the (generalized weak) $\Phi$-functions and relate them with other assumptions in the literature. We conclude by proving two auxiliary results, Lemmas 2.11 and 2.12. The first one can be interpreted as a counterpart in our setting of the weighted power-mean inequality for the function $\varphi(x, t)=t^{p}$ for some $p \geqslant 1$; the second one is a Jensen-type inequality in the spirit of [22, Lemma 4.4] and [24, Lemma 2.2].

2.1. Notation. We denote by $\mathbb{R}^{n}$ the $n$-dimensional real Euclidean space. We write $B(x, r)$ for the open ball in $\mathbb{R}^{n}$ centered at $x \in \mathbb{R}^{n}$ and with radius $r>0$. We use $c$ as a generic positive constant; i.e., a constant whose value may change from appearance to appearance. If $E \subset \mathbb{R}^{n}$ is a measurable set, then $|E|$ stands for its (Lebesgue) measure and $\chi_{E}$ denotes its characteristic function; we denote by $L^{0}(E)$ the space of all Lebesgue measurable functions on $E$.

Let $g, h: D \subset \mathbb{R}^{m} \rightarrow[0, \infty]$ be two functions. We write $g \lesssim h$ to mean that there exists a positive constant, $C$, such that $g(z) \leqslant C h(z)$ for all $z \in D$. If $g \lesssim h \lesssim g$, we write $g \approx h$. Also, given a sequence $\left(g_{\varepsilon}\right)_{\varepsilon}$ of non-negative functions in $D$, the notation $\lim _{\varepsilon \rightarrow 0} g_{\varepsilon} \approx h$ means that there is a positive constant, $C$, such that $\frac{1}{C} h \leqslant \liminf _{\varepsilon \rightarrow 0} g_{\varepsilon} \leqslant$ $\lim \sup _{\varepsilon \rightarrow 0} g_{\varepsilon} \leqslant C h$ in $D$; i.e., for the equivalence we do not require the limit to exist, only the upper and lower limits to be within a constant of each other.

Moreover, if $D=[0, \infty)$, we say that $g$ and $h$ are equivalent, written $g \simeq h$, if there exists $L \geqslant 1$ such that for all $t \geqslant 0$, we have $h\left(\frac{t}{L}\right) \leqslant g(t) \leqslant h(L t)$. In this case, $L$ is said to be the equivalence constant.

Let $\varphi:[0, \infty) \rightarrow[0, \infty]$ be an increasing function. We denote by $\varphi^{-1}:[0, \infty] \rightarrow[0, \infty]$ the left-continuous generalized inverse of $\varphi$; that is, for all $s \in[0, \infty]$,

$$
\varphi^{-1}(s):=\inf \{t \geqslant 0 \mid \varphi(t) \geqslant s\} .
$$

Let $\Omega \subset \mathbb{R}^{n}$ be open, let $\varphi: \Omega \times[0, \infty) \rightarrow[0, \infty]$, and let $B \subset \mathbb{R}^{n}$. Then, $\varphi_{B}^{+}:[0, \infty) \rightarrow$ $[0, \infty]$ and $\varphi_{B}^{-}:[0, \infty) \rightarrow[0, \infty]$ are the functions defined for all $t \in[0, \infty)$ by

$$
\varphi_{B}^{+}(t):=\sup _{x \in \Omega \cap B} \varphi(x, t) \text { and } \varphi_{B}^{-}(t):=\inf _{x \in \Omega \cap B} \varphi(x, t) .
$$

Note that if $\varphi(x, \cdot)$ is increasing for every $x \in \Omega$, then so are $\varphi_{B}^{+}(\cdot)$ and $\varphi_{B}^{-}(\cdot)$; thus, these functions admit a left-continuous generalized inverse in the sense of (2.1).

2.2. $\Phi$-FunCtions AND GENERALIZED ORLICZ SPACES. We start this subsection by introducing the notion of almost increasing and almost decreasing functions, after which we recall the definition of $\Phi$-functions and of some of its generalizations.

Definition 2.1. We say that a function $g: D \subset \mathbb{R} \rightarrow[0, \infty]$ is almost increasing if $g\left(t_{1}\right) \leqslant c g\left(t_{2}\right)$ for every $t_{1} \leqslant t_{2}$ in $D$ and some $c$. We say that $c$ is the monotonicity constant of $g$. Almost decreasing is defined analogously. 
Definition 2.2. Let $\varphi:[0, \infty) \rightarrow[0, \infty]$ be an increasing function satisfying $\varphi(0)=$ $\lim _{t \rightarrow 0^{+}} \varphi(t)=0$ and $\lim _{t \rightarrow \infty} \varphi(t)=\infty$. We say that $\varphi$ is a

(i) weak $\Phi$-function if $t \mapsto \frac{\varphi(t)}{t}$ is almost increasing.

(ii) $\Phi$-function if it is left-continuous and convex.

We denote by $\Phi_{w}$ the set of all weak $\Phi$-functions and by $\Phi$ the set of all $\Phi$-functions.

Remark 2.3. If $\varphi \in \Phi$, then, by convexity and because $\varphi(0)=0$, for $0<t_{1}<t_{2}$, $\varphi\left(t_{1}\right)=\varphi\left(\frac{t_{1}}{t_{2}} t_{2}+\left(1-\frac{t_{1}}{t_{2}}\right) 0\right) \leqslant \frac{t_{1}}{t_{2}} \varphi\left(t_{2}\right)$; thus, $t \mapsto \frac{\varphi(t)}{t}$ is increasing. Hence, $\Phi \subset \Phi_{w}$. Conversely, if $\varphi \in \Phi_{w}$, then there exists $\psi \in \Phi$ such that $\varphi \simeq \psi$ [19, Proposition 2.3].

Definition 2.4. Let $\Omega \subset \mathbb{R}^{n}$ be an open set, and let $\varphi: \Omega \times[0, \infty) \rightarrow[0, \infty]$ be a function such that $\varphi(\cdot, t) \in L^{0}(\Omega)$ for every $t \in[0, \infty)$. We say that $\varphi$ is a

(i) generalized weak $\Phi$-function on $\Omega$ if $\varphi(x, \cdot) \in \Phi_{w}$ uniformly in $x \in \Omega$; i.e., the monotonicity constant is independent of $x$.

(ii) generalized $\Phi$-function on $\Omega$ if $\varphi(x, \cdot) \in \Phi$ for every $x \in \Omega$.

We denote by $\Phi_{w}(\Omega)$ and $\Phi(\Omega)$ the sets of generalized weak $\Phi$-functions and generalized $\Phi$-functions, respectively.

By this definition, it is clear that properties of (weak) $\Phi$-functions carry over to generalized (weak) $\Phi$-functions point-wise uniformly. In particular, this holds for Remark 2.3. Similarly, $\varphi \simeq \psi$ means that $\varphi(x, \cdot) \simeq \psi(x, \cdot)$ with constant uniform in $x$, etc.

Next, we recall the definition of the generalized Orlicz space, quasi-norm, and quasisemimodular associated with a generalized weak $\Phi$-function.

Definition 2.5. Let $\Omega \subset \mathbb{R}^{n}$ be an open set, let $\varphi \in \Phi_{w}(\Omega)$, and consider the weak quasi-semimodular, $\varrho_{\varphi(\cdot), \Omega}$, on $L^{0}(\Omega)$ defined by

$$
\varrho_{\varphi(\cdot), \Omega}(f):=\int_{\Omega} \varphi(x,|f(x)|) d x
$$

for all $f \in L^{0}(\Omega)$. The generalized Orlicz space, $L^{\varphi(\cdot)}(\Omega)$, is given by

$$
L^{\varphi(\cdot)}(\Omega):=\left\{f \in L^{0}(\Omega) \mid \varrho_{\varphi(\cdot), \Omega}(\lambda f)<\infty \text { for some } \lambda>0\right\} .
$$

We endow $L^{\varphi(\cdot)}(\Omega)$ with the quasi-norm

$$
\|f\|_{\varphi(\cdot), \Omega}:=\inf \left\{\lambda>0 \mid \varrho_{\varphi(\cdot), \Omega}(f / \lambda) \leqslant 1\right\} .
$$

If, in the Definition 2.5, $\varphi \in \Phi(\Omega)$, then $\varrho_{\varphi(\cdot), \Omega}(\cdot)$ defines a semimodular on $L^{0}(\Omega)$ and $\|\cdot\|_{\varphi(\cdot), \Omega}$ a norm on $L^{\varphi(\cdot)}(\Omega)$ (see $\left.[14]\right)$.

Remark 2.6. If $\varphi, \psi \in \Phi_{w}(\Omega)$ and $\varphi \simeq \psi$, then $L^{\varphi(\cdot)}=L^{\psi(\cdot)}$ with equivalent quasinorms.

2.3. MAin ASSUMPtions. We begin by introducing our main assumptions on the generalized (weak) $\Phi$-functions on $\Omega$. The first three assumptions, $\left(\Delta_{2}\right)$, (aInc), and (aDec), extend three known properties for $\Phi$-functions to generalized $\Phi$-functions point-wise uniformly. The fourth assumption, (A), relates the behavior of generalized $\Phi$-functions at different values of the variable in $\Omega$. The last assumption, (loc), implies that simple functions belong to the generalized Orlicz space. These last two assumptions hold trivially for Orlicz spaces.

Let $\Omega \subset \mathbb{R}^{n}$ be open and $\varphi \in \Phi_{w}(\Omega)$. We denote by $\varphi^{-1}$ the generalized inverse of $\varphi$ with respect to the second variable (see (2.1)). 
$\left(\Delta_{2}\right) \varphi$ is doubling; i.e., there exists $A>0$ such that $\varphi(x, 2 t) \leqslant A \varphi(x, t)$ for a.e. $x \in \Omega$ and for all $t \geqslant 0$.

(aInc) There exists a constant $\varphi_{\uparrow}>1$ such that for a.e. $x \in \Omega$, the map $s \mapsto s^{-\varphi_{\uparrow}} \varphi(x, s)$ is almost increasing with monotonicity constant $c_{\uparrow}$ independent of $x$.

(aDec) There exists a constant $\varphi_{\downarrow}>1$ such that for a.e. $x \in \Omega$, the map $s \mapsto s^{-\varphi_{\downarrow}} \varphi(x, s)$ is almost decreasing with monotonicity constant $c_{\downarrow}$ independent of $x$.

(A) There exist $\beta, \sigma>0$ for which:

(A0) $\varphi(x, \beta \sigma) \leqslant 1 \leqslant \varphi(x, \sigma)$ for all $x \in \Omega$;

(A1) $\varphi(x, \beta t) \leqslant \varphi(y, t)$ for every ball $B \subset \Omega, x, y \in B$, and $t \in\left[\sigma, \varphi^{-1}\left(y, \frac{1}{|B|}\right)\right]$;

(A2) there exists $h \in L^{1}(\Omega) \cap L^{\infty}(\Omega)$ such that for a.e. $x, y \in \Omega$ and for all $t \in[0, \sigma]$, we have $\varphi(x, \beta t) \leqslant \varphi(y, t)+h(x)+h(y)$.

(loc) There exists $t_{0}>0$ such that $\varphi\left(\cdot, t_{0}\right) \in L_{\text {loc }}^{1}(\Omega)$.

The notation (aInc) $)_{1}$ is used for a version of (aInc) with $\varphi_{\uparrow} \geqslant 1$; i.e., equality included. Note that, for any weak $\Phi$-function, (aInc) ${ }_{1}$ holds for $\varphi_{\uparrow}=1$.

Remark 2.7. Let us collect several observations regarding the assumptions above.

1. Each of the previous conditions is invariant under equivalence of (weak) $\Phi$-functions; i.e., if $\varphi \simeq \psi$, then $\varphi$ satisfies a condition if and only if $\psi$ satisfies it.

2 . For doubling (weak) $\Phi$-functions, $\simeq$ and $\approx$ are equivalent.

3. $(\mathrm{aDec})$ and $\left(\Delta_{2}\right)$ are equivalent [21, Lemma 2.6].

4. (A0) implies (loc) (choose $t_{0}:=\beta \sigma$ ).

5. If (aInc) and (aDec) hold, then $\varphi^{\uparrow} \leqslant \varphi^{\downarrow}$.

6. Finally, note that if $\varphi$ satisfies (aDec) and (loc), then $\varphi(\cdot, t) \in L_{\text {loc }}^{1}(\Omega)$ for every $t \geqslant 0$.

It follows directly from the definition of the left-inverse that $\varphi \simeq \psi$ implies $\varphi^{-1} \approx \psi^{-1}$. Furthermore, if $\varphi \simeq \psi$, then $\varphi_{B}^{-} \simeq \psi_{B}^{-}$and so $\left(\varphi_{B}^{-}\right)^{-1} \approx\left(\psi_{B}^{-}\right)^{-1}$.

The next proposition shows that Assumption (A1) is equivalent to its counterpart in [22].

Lemma 2.8. Let $\varphi \in \Phi_{w}(\Omega), \sigma>0$, and $\beta \in(0,1)$. Then, $\varphi$ satisfies (A1) for $(\sigma, \beta)$ if and only if for every ball $B \subset \Omega$, and for all finite $t \in\left[\sigma,\left(\varphi_{B}^{-}\right)^{-1}(1 /|B|)\right]$, we have

$$
\varphi_{B}^{+}(\beta t) \leqslant \varphi_{B}^{-}(t) .
$$

Proof. Because $\left(\varphi_{B}^{-}\right)^{-1}\left(\frac{1}{|B|}\right) \geqslant \varphi^{-1}\left(y, \frac{1}{|B|}\right)$, it follows that if $\varphi$ satisfies $(2.2)$, then it also satisfies (A1).

Conversely, assume that $\varphi$ satisfies (A1). We first consider the case when $t \in\left[\sigma,\left(\varphi_{B}^{-}\right)^{-1}\left(\frac{1}{|B|}\right)\right)$. In this case, we can find $\left(y_{i}\right)_{i \in \mathbb{N}} \subset B$ such that $t \in\left[\sigma, \varphi^{-1}\left(y_{i}, \frac{1}{|B|}\right)\right]$ for all $i \in \mathbb{N}$ and $\varphi_{B}^{-}(t)=\lim _{i \rightarrow \infty} \varphi\left(y_{i}, t\right)$. Then, by (A1), we have

$$
\varphi(x, \beta t) \leqslant \varphi\left(y_{i}, t\right)
$$

for a.e. $x \in B$ and for all $i \in \mathbb{N}$. Taking the supremum over $x \in B$ and then letting $i \rightarrow \infty$ in the previous estimate, we obtain $\varphi_{B}^{+}(\beta t) \leqslant \varphi_{B}^{-}(t)$. Finally, assume that $t=$ $\left(\varphi_{B}^{-}\right)^{-1}\left(\frac{1}{|B|}\right)<\infty$, and let $t^{\prime} \in[\sigma, t)$. By the previous case,

$$
\varphi\left(x, \beta t^{\prime}\right) \leqslant \varphi_{B}^{+}\left(\beta t^{\prime}\right) \leqslant \varphi_{B}^{-}\left(t^{\prime}\right) \leqslant \varphi_{B}^{-}(t)
$$

for a.e. $x \in B$, where in the last inequality we used the fact that $\varphi_{B}^{-}$is increasing. Taking the limit $t^{\prime} \rightarrow t$ in $(2.3)$, the left-continuity of $\varphi(x, \cdot)$ yields $\varphi(x, \beta t) \leqslant \varphi_{B}^{-}(t)$ for a.e. $x \in B$. Hence, taking the supremum over $x \in B$, we conclude that $\varphi$ satisfies (2.2). 
The next lemma shows that left-inverse commutes with infimum, even when the function is not continuous.

Lemma 2.9. Let $\Omega \subset \mathbb{R}^{n}$ be an open set, let $B \subset \Omega$, and let $\varphi \in \Phi_{w}(\Omega)$. Then, $\left(\varphi_{B}^{-}\right)^{-1}=\left(\varphi^{-1}\right)_{B}^{+}$.

Proof. Fix $s \in[0, \infty]$. For every $x \in B$, we have

$$
\left(\varphi_{B}^{-}\right)^{-1}(s)=\inf \left\{t \geqslant 0 \mid \varphi_{B}^{-}(t) \geqslant s\right\} \geqslant \inf \{t \geqslant 0 \mid \varphi(x, t) \geqslant s\}=\varphi^{-1}(x, s) .
$$

Thus, taking the supremum over $x \in B$,

$$
\left(\varphi_{B}^{-}\right)^{-1}(s) \geqslant\left(\varphi^{-1}\right)_{B}^{+}(s) .
$$

To prove the converse inequality, we may assume that $\bar{t}:=\left(\varphi^{-1}\right)_{B}^{+}(s)<\infty$ without loss of generality. Fix $\varepsilon>0$. By definition of $\bar{t}$ and because $\varphi(x, \cdot)$ is increasing for every $x \in B$, we have $\varphi(x, \bar{t}+\varepsilon) \geqslant s$ for all $x \in B$. Hence, $\left(\varphi_{B}^{-}\right)^{-1}(s) \leqslant \bar{t}+\varepsilon$. Letting $\varepsilon \rightarrow 0$, we conclude the desired inequality.

The following lemma allows us to relate Assumption (A1) with its counterpart in [18].

Lemma 2.10. Let $\Omega \subset \mathbb{R}^{n}$ be open and assume that $\varphi \in \Phi(\Omega)$ is doubling and satisfies (A0). Then, (A1) is equivalent to the following condition:

$\left(A 1^{\prime}\right) \varphi^{-1}(x, s) \lesssim \varphi^{-1}(y, s)$ for every ball $B \subset \Omega, x, y \in B$, and $s \in\left[1, \frac{1}{|B|}\right]$.

Proof. Because $\varphi$ belongs to $\Phi(\Omega)$ and is doubling, it is a bijection with respect to the second variable from $[0, \infty)$ to $[0, \infty)$. Applying $\varphi^{-1}$ to $(\mathrm{A} 0)$ and $(\mathrm{A} 1)$, we find that

$$
(\mathrm{A} 0) \Leftrightarrow \beta \sigma \leqslant \varphi^{-1}(x, 1) \leqslant \sigma \text { for a.e. } x \in \Omega \text {; }
$$

$$
(\mathrm{A} 1) \Leftrightarrow \beta \varphi^{-1}(x, s) \leqslant \varphi^{-1}(y, s) \text { for a.e. } x, y \in B \text { and for all } s \in\left[\varphi(x, \sigma), \frac{1}{|B|}\right] \text {. }
$$

If $s \in[1, \varphi(x, \sigma)]$, then $\beta \varphi^{-1}(x, 1) \leqslant \beta \varphi^{-1}(x, s) \leqslant \beta \sigma$ because $\varphi^{-1}(x, \cdot)$ is increasing. Thus, using $(2.4), \beta \varphi^{-1}(x, s) \leqslant \varphi^{-1}(y, s)$ holds for all such $s$.

As mentioned at the beginning of Section 2, the following lemma can be interpreted as a counterpart in our setting of the weighted power-mean inequality for the function $\varphi(x, t)=t^{p}$ for some $p \geqslant 1$.

Lemma 2.11. Let $\Omega \subset \mathbb{R}^{n}$ be open and assume that $\varphi \in \Phi_{w}(\Omega)$ satisfies (aDec). Then, for all $\delta>0$ and $a, b \geqslant 0$ and for a.e. $x \in \Omega$, we have

$$
\varphi(x, a+b) \leqslant \varphi(x,(1+\delta) a)+\frac{1}{c_{\downarrow}}\left(1+\frac{1}{\delta}\right)^{\varphi_{\downarrow}} \varphi(x, b)
$$

and

$$
\varphi(x, a+b) \leqslant \frac{1}{c_{\downarrow}}\left[(1+\delta)^{\varphi_{\downarrow}} \varphi(x, a)+\left(1+\frac{1}{\delta}\right)^{\varphi_{\downarrow}} \varphi(x, b)\right] .
$$

Proof. If $b \leqslant \delta a$, then the monotonicity of $\varphi(x, \cdot)$ yields

$$
\varphi(x, a+b) \leqslant \varphi(x,(1+\delta) a) .
$$

If $a<\delta^{-1} b$, then the monotonicity of $\varphi(x, \cdot)$ and (aDec) yield

$$
\varphi(x, a+b) \leqslant \varphi\left(x,\left(1+\delta^{-1}\right) b\right) \leqslant \frac{1}{c_{\downarrow}}\left(1+\delta^{-1}\right)^{\varphi_{\downarrow}} \varphi(x, b) .
$$

Thus, (2.5) holds. Further, (2.6) follows from (2.5) by (aDec). 
The following lemma is a variant of [22, Lemma 4.4] without the assumption $\rho_{\varphi(\cdot)}\left(f \chi_{\{|f|>\sigma\}}\right)<$ 1 and correspondingly weaker conclusion (see also [24, Lemma 2.2]).

Lemma 2.12. Let $\Omega \subset \mathbb{R}^{n}$ be open and assume that $\varphi \in \Phi_{w}(\Omega)$ satisfies Assumption (A). Then, there exists $\beta^{\prime}>0$ such that, for every ball $B \subset \Omega, f \in L^{\varphi(\cdot)}(\Omega)$, and a.e. $x \in B$, we have

$$
\varphi\left(x, \beta^{\prime} \min \left\{\left(\varphi_{B}^{-}\right)^{-1}\left(\frac{1}{|B|}\right), f_{B}|f(y)| d y\right\}\right) \leqslant f_{B} \varphi(y,|f(y)|) d y+h(x)+f_{B} h(y) d y,
$$

where $h$ is the function provided by (A2).

Proof. Without loss of generality, we may assume that $f \geqslant 0$. By Remarks 2.3 and 2.7, we may also assume that $\varphi \in \Phi(\Omega)$. Then, $t \mapsto \frac{\varphi(x, t)}{t}$ is increasing for every $x \in \Omega$.

Fix a ball $B \subset \Omega$, and denote $\alpha:=\left(\varphi_{B}^{-}\right)^{-1}\left(\frac{1}{|B|}\right)$. Let $(\sigma, \beta)$ be given by Assumption (A), and set $f_{1}:=f \chi_{\{f>\sigma\}}, f_{2}:=f-f_{1}$, and $F_{i}:=f_{B} f_{i} d y$ for $i \in\{1,2\}$. Because $\varphi(x, \cdot)$ is convex and increasing,

$$
\varphi\left(x, \frac{\beta}{4} \min \left\{\alpha, f_{B} f d y\right\}\right) \leqslant \varphi\left(x, \frac{\beta}{2} \min \left\{\alpha, F_{1}\right\}\right)+\varphi\left(x, \beta F_{2}\right) .
$$

We start by estimating the first term on the right-hand side of (2.7). Suppose first that $\frac{1}{2} \min \left\{\alpha, F_{1}\right\}>\sigma$. Then, by definition of $\alpha, \frac{1}{2} \min \left\{\alpha, F_{1}\right\} \in\left[\sigma,\left(\varphi_{B}^{-}\right)^{-1}\left(\frac{1}{|B|}\right)\right]$. Thus, Lemma 2.8 and the monotonicity of $\varphi(x, \cdot)$ and $\varphi_{B}^{-}$yield $\varphi\left(x, \frac{\beta}{2} \min \left\{\alpha, F_{1}\right\}\right) \leqslant \varphi_{B}^{-}\left(\frac{1}{2} F_{1}\right)$. Using now [22, Lemma 4.3], we obtain

$$
\varphi\left(x, \frac{\beta}{2} \min \left\{\alpha, F_{1}\right\}\right) \leqslant \varphi_{B}^{-}\left(\frac{1}{2} F_{1}\right) \leqslant f_{B} \varphi_{B}^{-}\left(f_{1}(y)\right) d y \leqslant f_{B} \varphi\left(y, f_{1}(y)\right) d y .
$$

Next, suppose that $\frac{1}{2} \min \left\{\alpha, F_{1}\right\} \leqslant \sigma$. By convexity and monotonicity of $\varphi(x, \cdot)$, by (A0), and by convexity again, we conclude that

$$
\varphi\left(x, \frac{\beta}{2} \min \left\{\alpha, F_{1}\right\}\right) \leqslant \varphi(x, \beta \sigma) \frac{F_{1}}{2 \sigma} \leqslant \frac{1}{2 \sigma} f_{B} f_{1}(y) d y \leqslant \frac{1}{2} f_{B} \varphi\left(y, f_{1}(y)\right) d y,
$$

where, in the last inequality, we used also (A0) together with the fact that $f_{1}(y)>\sigma$ in $\left\{y \in \Omega: f_{1}(y) \neq 0\right\}$.

To estimate the second term on the right-hand side of (2.7), we invoke the convexity and monotonicity of $\varphi(x, \cdot)$ and Assumption (A2) to obtain

$$
\varphi\left(x, \beta F_{2}\right) \leqslant f_{B} \varphi\left(x, \beta f_{2}(y)\right) d y \leqslant f_{B} \varphi\left(y, f_{2}(y)\right) d y+h(x)+f_{B} h(y) d y .
$$

Recalling that $\alpha=\left(\varphi_{B}^{-}\right)^{-1}\left(\frac{1}{|B|}\right)$ and $\varphi(x, 0)=0$, the claim follows.

\section{Auxiliary Results}

Lemma 3.1. Let $\Omega$ and $U$ be two open subsets of $\mathbb{R}^{n}$ such that $U \subset \subset \Omega$. Let $\varphi \in \Phi_{w}(\Omega)$ satisfy Assumptions (aDec) and (loc), and let $\left(\psi_{\varepsilon}\right)_{\varepsilon}$ be a family of functions satisfying (1.1) and (1.2). Then,

$$
\lim _{\varepsilon \rightarrow 0^{+}} \varrho_{\#, U}^{\varepsilon}(f) \approx \varrho_{\varphi(\cdot), U}\left(c_{n}|\nabla f|\right)
$$

for all $f \in C^{\infty}(\Omega)$, where $c_{n}:=f_{B(0,1)}\left|x \cdot e_{1}\right| d x$. If, in addition, $\varphi(x, \cdot)$ is continuous for all $x \in U$, then (3.1) holds with equality.

Remark 3.2. Note that $\varphi(x, \cdot)$ is continuous for all $x \in \Omega$ if $\varphi \in \Phi(\Omega)$ satisfies (aDec). 
Proof. Let $f \in C^{\infty}(\Omega)$. We start by treating the case in which $\varphi(x, \cdot)$ is continuous for all $x \in U$. We claim that

$$
\lim _{\varepsilon \rightarrow 0^{+}} \varrho_{\#, U}^{\varepsilon}(f)=\varrho_{\varphi(\cdot), U}\left(c_{n}|\nabla f|\right) .
$$

By the Taylor expansion formula, for any $x \in \Omega$ and $y \in \Omega$ such that $|y-x|<$ $\operatorname{dist}(x, \partial \Omega)$, we have

$$
f(y)=f(x)+\nabla f(x) \cdot(y-x)+R(x, y),
$$

where $R(x, y)=o(|x-y|)$ as $y \rightarrow x$. Denote $h(x, r):=\frac{2}{r} f_{B(x, r)}|R(x, y)| d y$, for $r>0$ and $x \in \Omega_{r}$, and $c_{n}=f_{B(0,1)}\left|x \cdot e_{1}\right| d x$. As proved in [24, Lemma 3.1], we have the point-wise estimate

$$
c_{n} r|\nabla f(x)|-r h(x, r) \leqslant M_{B(x, r)}^{\#} f \leqslant c_{n} r|\nabla f(x)|+r h(x, r) .
$$

Consequently, we can define a function $\alpha: \Omega \times \mathbb{R}^{+} \rightarrow[-1,1]$ such that $\frac{1}{r} M_{B(x, r)}^{\#} f=$ $c_{n}|\nabla f(x)|+\alpha(x, r) h(x, r)$ when $x \in \Omega_{r}$. Then,

$$
\varrho_{\#, U}^{\varepsilon}(f)=\int_{0}^{\infty} \int_{U_{r}} \varphi\left(x, c_{n}|\nabla f(x)|+\alpha(x, r) h(x, r)\right) d x \psi_{\varepsilon}(r) d r .
$$

Next, we prove that $\limsup _{\varepsilon \rightarrow 0^{+}} \varrho_{\#, U}^{\varepsilon}(f) \leqslant \varrho_{\varphi(\cdot), U}\left(c_{n}|\nabla f|\right)$. Fix $\delta>0$. Because $c_{n}|\nabla f(x)|+\alpha(x, r) h(x, r) \leqslant c_{n}|\nabla f(x)|+h(x, r)$, the monotonicity of $\varphi$ and (2.5) yield

$$
\varphi\left(x, c_{n}|\nabla f(x)|+\alpha(x, r) h(x, r)\right) \leqslant \varphi\left(x,(1+\delta) c_{n}|\nabla f(x)|\right)+\frac{1}{c_{\downarrow}}\left(1+\frac{1}{\delta}\right)^{\varphi_{\downarrow}} \varphi(x, h(x, r)) .
$$

Hence, invoking (3.3) and (1.1), we obtain

$$
\begin{aligned}
\limsup _{\varepsilon \rightarrow 0^{+}} \varrho_{\#, U}^{\varepsilon}(f) \leqslant & \varrho_{\varphi(\cdot), U}\left((1+\delta) c_{n}|\nabla f|\right) \\
& +\frac{1}{c_{\downarrow}}\left(1+\frac{1}{\delta}\right)^{\varphi_{\downarrow}} \limsup _{\varepsilon \rightarrow 0^{+}} \int_{0}^{\infty} \int_{U_{r}} \varphi(x, h(x, r)) d x \psi_{\varepsilon}(r) d r .
\end{aligned}
$$

We claim that

$$
\lim _{\varepsilon \rightarrow 0^{+}} \int_{0}^{\infty} \int_{U_{r}} \varphi(x, h(x, r)) d x \psi_{\varepsilon}(r) d r=0
$$

from which the estimate on the upper limit follows by dominated convergence as $\delta \rightarrow 0^{+}$ in (3.4) taking also into account the continuity of $\varphi(x, \cdot)$.

To prove (3.5), we start by observing that because $U$ is bounded, the set $U_{r}$ is empty for all $r>0$ sufficiently large. Thus, there exists $r_{0}>0$ for which we have

$$
\int_{0}^{\infty} \int_{U_{r}} \varphi(x, h(x, r)) d x \psi_{\varepsilon}(r) d r=\int_{0}^{r_{0}} \int_{U_{r}} \varphi(x, h(x, r)) d x \psi_{\varepsilon}(r) d r .
$$

Moreover, since $f \in C^{2},|R(x, y)| \leqslant\|f\|_{W^{2, \infty}(U)}|x-y|^{2}$ for all $r \in\left(0, r_{0}\right), x \in U_{r}$, and $y \in B(x, r)$. Set $C:=\|f\|_{W^{2, \infty}(U)}$. Then, for all $r \in\left(0, r_{0}\right)$ and $x \in U_{r}, h(x, r) \leqslant$ $2 C r$. Fix $0<\gamma<\min \left\{1, r_{0}\right\}$. We have that $\varphi(x, h(x, r)) \leqslant \varphi\left(x, 2 C r_{0}\right)$ whenever $r \in$ $\left(\gamma, r_{0}\right)$; moreover, denoting by $c$ the monotonicity constant of $t \mapsto \frac{\varphi(x, t)}{t}$, we have also $\varphi(x, h(x, r)) \leqslant c \gamma \varphi(x, 2 C)$ whenever $r \in(0, \gamma]$. Using, in addition, the condition $\varphi \geqslant 0$ and (1.1), it follows that

$$
\int_{0}^{r_{0}} \int_{U_{r}} \varphi(x, h(x, r)) d x \psi_{\varepsilon}(r) d r \leqslant c \gamma \int_{U} \varphi(x, 2 C) d x+\int_{U} \varphi\left(x, 2 C r_{0}\right) d x \int_{\gamma}^{\infty} \psi_{\varepsilon}(r) d r .
$$

In view of (1.2) and Assumption (loc), together with Remark 2.7, letting $\varepsilon \rightarrow 0^{+}$in this estimate first, and then $\gamma \rightarrow 0^{+}$, we obtain (3.5). 
Finally, we prove that $\liminf _{\varepsilon \rightarrow 0^{+}} \varrho_{\#, U}^{\varepsilon}(f) \geqslant \varrho_{\varphi(\cdot), U}\left(c_{n}|\nabla f|\right)$. Fix $\delta>0$, and denote $a^{\prime}:=c_{n}|\nabla f(x)|$ and $b^{\prime}:=\alpha(x, r) h(x, r)$. If $r>0$ and $x \in U_{r}$ are such that $\alpha(x, r)<0$, then applying (2.5) with $a:=\frac{a^{\prime}+b^{\prime}}{1+\delta}$ and $b:=-\frac{b^{\prime}}{1+\delta}$ gives

$$
\begin{aligned}
\varphi\left(x, c_{n}|\nabla f(x)|+\alpha(x, r) h(x, r)\right) & =\varphi(x,(1+\delta) a) \geqslant \varphi(x, a+b)-\frac{1}{c_{\downarrow}}\left(1+\frac{1}{\delta}\right)^{\varphi_{\downarrow}} \varphi(x, b) \\
& \geqslant \varphi\left(x, \frac{c_{n}|\nabla f(x)|}{1+\delta}\right)-\frac{1}{c_{\downarrow}}\left(1+\frac{1}{\delta}\right)^{\varphi_{\downarrow}} \varphi(x, h(x, r)),
\end{aligned}
$$

where we used the monotonicity of $\varphi$ and the estimate $0 \leqslant-\frac{b^{\prime}}{1+\delta} \leqslant h(x, r)$. If $r>0$ and $x \in U_{r}$ are such that $\alpha(x, r) \geqslant 0$, then the monotonicity of $\varphi$ yields

$$
\varphi\left(x, c_{n}|\nabla f(x)|+\alpha(x, r) h(x, r)\right) \geqslant \varphi\left(x, \frac{c_{n}|\nabla f(x)|}{1+\delta}\right) .
$$

Thus, by (3.6) and (3.7), for every $r>0$ and $x \in U_{r}$, we have

$$
\varphi\left(x, c_{n}|\nabla f(x)|+\alpha(x, r) h(x, r)\right) \geqslant \varphi\left(x, \frac{c_{n}}{1+\delta}|\nabla f(x)|\right)-\frac{1}{c_{\downarrow}}\left(1+\frac{1}{\delta}\right)^{\varphi_{\downarrow}} \varphi(x, h(x, r)) .
$$

Consequently, by (3.3), it follows that

$$
\varrho_{\#, U}^{\varepsilon}(f) \geqslant \int_{0}^{\infty} \int_{U_{r}}\left[\varphi\left(x, \frac{c_{n}}{1+\delta}|\nabla f(x)|\right)-\frac{1}{c_{\downarrow}}\left(1+\frac{1}{\delta}\right)^{\varphi_{\downarrow}} \varphi(x, h(x, r))\right] d x \psi_{\varepsilon}(r) d r .
$$

Fix $0<\gamma<1$ and $r_{0}>0$ such that $U_{r_{0}} \neq \emptyset$. By (1.1) and (1.2), there exists $\varepsilon_{0}=\varepsilon_{0}\left(\gamma, r_{0}\right)$ such that, for all $\varepsilon \in\left(0, \varepsilon_{0}\right)$, we have

$$
\int_{0}^{r_{0}} \psi_{\varepsilon}(r) d r \geqslant 1-\gamma
$$

Thus, we have also, for $0<\varepsilon<\varepsilon_{0}$,

$$
\begin{aligned}
\int_{0}^{\infty} \int_{U_{r}} \varphi\left(x, \frac{c_{n}}{1+\delta}|\nabla f(x)|\right) d x \psi_{\varepsilon}(r) d r & \geqslant \int_{0}^{r_{0}} \int_{U_{r_{0}}} \varphi\left(x, \frac{c_{n}}{1+\delta}|\nabla f(x)|\right) d x \psi_{\varepsilon}(r) d r \\
& \geqslant(1-\gamma) \int_{U_{r_{0}}} \varphi\left(x, \frac{c_{n}}{1+\delta}|\nabla f(x)|\right) d x .
\end{aligned}
$$

This estimate, (3.8), and (3.5) yield

$$
\liminf _{\varepsilon \rightarrow 0^{+}} \varrho_{\#, U}^{\varepsilon}(f) \geqslant(1-\gamma) \varrho_{\varphi(\cdot), U_{r_{0}}}\left(\frac{c_{n}}{1+\delta}|\nabla f|\right) .
$$

The conclusion then follows by using the continuity of $\varphi(x, \cdot)$ and by letting $\delta \rightarrow 0^{+}$, $\gamma \rightarrow 0^{+}$, and $r_{0} \rightarrow 0^{+}$in this order. This completes the proof of (3.2) under the continuity assumption.

Suppose now that $\varphi$ is a general weak $\Phi$-function. By Remarks 2.3 and 2.7, there exists $\psi \in \Phi(\Omega)$ satisfying the same assumptions as $\varphi$ and such that $\psi \approx \varphi$. Then, there is $C>0$ such that $\frac{1}{C} \varphi \leqslant \psi \leqslant C \varphi$. Hence, by the first part of the proof,

$$
\begin{aligned}
& \frac{1}{C^{2}} \varrho_{\varphi(\cdot), U}\left(c_{n}|\nabla f|\right) \leqslant \frac{1}{C} \varrho_{\psi(\cdot), U}\left(c_{n}|\nabla f|\right)=\frac{1}{C} \liminf _{\varepsilon \rightarrow 0^{+}} \varrho_{\#, U, \psi}^{\varepsilon}(f) \leqslant \liminf _{\varepsilon \rightarrow 0^{+}} \varrho_{\#, U, \varphi}^{\varepsilon}(f) \\
& \quad \leqslant \limsup _{\varepsilon \rightarrow 0^{+}} \varrho_{\#, U, \varphi}^{\varepsilon}(f) \leqslant C \limsup _{\varepsilon \rightarrow 0^{+}} \varrho_{\#, U, \psi}^{\varepsilon}(f)=C \varrho_{\psi(\cdot), U}\left(c_{n}|\nabla f|\right) \leqslant C^{2} \varrho_{\varphi(\cdot), U}\left(c_{n}|\nabla f|\right) .
\end{aligned}
$$

Next, we derive an auxiliary upper bound which holds for all functions $f \in L_{\text {loc }}^{1}(\Omega)$ with $|\nabla f| \in L^{\varphi(\cdot)}(\Omega)$.

Lemma 3.3. Let $\Omega$ be an open set of $\mathbb{R}^{n}$, let $\varphi \in \Phi_{w}(\Omega)$ satisfy Assumptions (A) and (aDec), and let $\left(\psi_{\varepsilon}\right)_{\varepsilon}$ be a family of functions satisfying (1.1) and (1.2). If $f \in L_{\mathrm{loc}}^{1}(\Omega)$ and $|\nabla f| \in L^{\varphi(\cdot)}(\Omega)$, then

$$
\varrho_{\#, \Omega}^{\varepsilon}(f) \leqslant c \max \left\{\|\nabla f\|_{\varphi(\cdot), \Omega}^{\varphi_{\uparrow}},\|\nabla f\|_{\varphi(\cdot), \Omega}^{\varphi_{\downarrow}}\right\} .
$$


Proof. By Remarks 2.3, 2.6, and 2.7 we may assume without loss of generality that $\varphi \in$ $\Phi(\Omega)$. Then, in view of Assumptions (A0) and (aInc) ${ }_{1}$, we have $L^{\varphi(\cdot)}(B) \subset L^{1}(B)$ for every bounded set $B \subset \Omega$ by [19, Lemma 4.4].

Let $f \in L_{\text {loc }}^{1}(\Omega)$ with $|\nabla f| \in L^{\varphi(\cdot)}(\Omega)$ be such that $\|\nabla f\|_{\varphi(\cdot), \Omega} \leqslant 1$. Fix $r>0$ and $x \in \Omega_{r}$. By the Poincaré inequality in $L^{1}$, we have

$$
\frac{1}{r} M_{B(x, r)}^{\#} f=\frac{1}{r} f_{B(x, r)}\left|f(y)-f_{B(x, r)}\right| d y \leqslant c f_{B(x, r)}|\nabla f(y)| d y .
$$

On the other hand, in view of Lemma 2.8, we may invoke [23, Lemma 4.4] (with $\gamma=1$ ) that gives the existence of a constant, $\beta^{\prime} \in(0,1)$, depending only on the constants in $(\mathrm{A})$, such that

$$
\varphi\left(x, \beta^{\prime} f_{B(x, r)}|\nabla f(y)| d y\right) \leqslant f_{B(x, r)} \varphi(y,|\nabla f(y)|) d y+h(x)+f_{B(x, r)} h(y) d y .
$$

Using the monotonicity of $\varphi$, (aDec), (3.9), and (3.10), we obtain

$$
\begin{aligned}
\varrho_{\#, \Omega}^{\varepsilon}(f) & \lesssim \int_{0}^{\infty} \int_{\Omega_{r}} \varphi\left(x, \beta^{\prime} f_{B(x, r)}|\nabla f(y)| d y\right) d x \psi_{\varepsilon}(r) d r \\
& \leqslant \int_{0}^{\infty} \int_{\Omega_{r}}\left(f_{B(x, r)} \varphi(y,|\nabla f(y)|) d y+h(x)+f_{B(x, r)} h(y) d y\right) d x \psi_{\varepsilon}(r) d r .
\end{aligned}
$$

Next, by changing the order of integration, we observe that

$$
\begin{aligned}
\int_{\Omega_{r}} f_{B(x, r)} \varphi(y,|\nabla f(y)|) d y d x & =\int_{\Omega_{r}} \int_{\Omega} \varphi(y,|\nabla f(y)|) \frac{\chi_{B(y, r)}(x)}{|B(x, r)|} d y d x \\
& \leqslant \int_{\Omega} \varphi(y,|\nabla f(y)|) d y=\varrho_{\varphi(\cdot), \Omega}(|\nabla f|) \leqslant 1 .
\end{aligned}
$$

Similarly,

$$
\int_{\Omega_{r}} f_{B(x, r)} h(y) d y d x \leqslant \int_{\Omega} h(y) d y .
$$

Consequently, because $h \in L^{1}(\Omega)$ and $\int_{0}^{\infty} \psi_{\varepsilon}(r) d r=1$, from (3.11) we conclude that

$$
\varrho_{\#, \Omega}^{\varepsilon}(f) \leqslant c \text { for all } f \in L_{\mathrm{loc}}^{1}(\Omega) \text { with }|\nabla f| \in L^{\varphi(\cdot)}(\Omega) \text { and }\|\nabla f\|_{\varphi(\cdot), \Omega} \leqslant 1 .
$$

By considering the cases $\lambda \leqslant 1$ and $\lambda>1$ and use Assumptions (aInc) 1 and (aDec), respectively, we find that $\varrho_{\#, \Omega}^{\varepsilon}(f) \leqslant c \varrho_{\#, \Omega}^{\varepsilon}\left(\frac{f}{\lambda}\right) \max \left\{\lambda^{\varphi_{\uparrow}}, \lambda^{\varphi_{\downarrow}}\right\}$ for all $\lambda>0$ and $f \in L_{\text {loc }}^{1}(\Omega)$ with $|\nabla f| \in L^{\varphi(\cdot)}(\Omega)$. Using this estimate with $\lambda:=\|\nabla f\|_{\varphi(\cdot), \Omega}+\delta$ for $\delta>0$ and then invoking (3.12), it follows that

$$
\begin{aligned}
\varrho_{\#, \Omega}^{\varepsilon}(f) & \leqslant c \varrho_{\#, \Omega}^{\varepsilon}\left(\frac{f}{\|\nabla f\|_{\varphi(\cdot), \Omega}+\delta}\right) \max \left\{\left(\|\nabla f\|_{\varphi(\cdot), \Omega}+\delta\right)^{\varphi_{\uparrow}},\left(\|\nabla f\|_{\varphi(\cdot), \Omega}+\delta\right)^{\varphi_{\downarrow}}\right\} \\
& \leqslant c \max \left\{\left(\|\nabla f\|_{\varphi(\cdot), \Omega}+\delta\right)^{\varphi_{\uparrow}},\left(\|\nabla f\|_{\varphi(\cdot), \Omega}+\delta\right)^{\varphi_{\downarrow}}\right\} .
\end{aligned}
$$

Letting $\delta \rightarrow 0^{+}$, we conclude the proof of Lemma 3.3 .

\section{MAIN RESUlts}

In this section we prove our main result, which provides a characterization of generalized Orlicz spaces. Theorem 1.1 is an immediate consequence of Propositions 4.1, 4.3 and 4.5 below. 
Proposition 4.1. Let $\Omega \subset \mathbb{R}^{n}$ be open, let $\varphi \in \Phi_{w}(\Omega)$ satisfy Assumptions (A), (aInc), and (aDec), and let $\left(\psi_{\varepsilon}\right)_{\varepsilon}$ be a family of functions satisfying (1.1) and (1.2). Assume that $f \in L_{\mathrm{loc}}^{1}(\Omega)$ with $|\nabla f| \in L^{\varphi(\cdot)}(\Omega)$. Then,

$$
\lim _{\varepsilon \rightarrow 0^{+}} \varrho_{\#, \Omega}^{\varepsilon}(f) \approx \varrho_{\varphi(\cdot), \Omega}\left(c_{n}|\nabla f|\right)<\infty
$$

where $c_{n}=f_{B(0,1)}\left|x \cdot e_{1}\right| d x$. If, in addition, $\varphi(x, \cdot)$ is continuous for all $x \in \Omega$, then (4.1) holds with equality.

Proof. We start with the upper bound. Let $U \subset \subset \Omega$. Using the same arguments as in [19, Theorems 6.5 and 6.6], we find $g_{\nu} \in C^{\infty}(\Omega), \nu \in \mathbb{N}$, such that $\left(\nabla g_{\nu}\right)_{n \in \mathbb{N}}$ converges to $\nabla f$ in $L^{\varphi(\cdot)}\left(U ; \mathbb{R}^{n}\right)$. For $r>0$ and $x \in U_{r}$, we have $M_{B(x, r)}^{\#} f \leqslant M_{B(x, r)}^{\#}\left(f-g_{\nu}\right)+M_{B(x, r)}^{\#} g_{\nu}$ by the triangle inequality. Combining this estimate with (2.5) applied to $a=\frac{1}{r} M_{B(x, r)}^{\#} g_{\nu}$ and $b=\frac{1}{r} M_{B(x, r)}^{\#}\left(f-g_{\nu}\right)$, we obtain

$$
\varrho_{\#, U}^{\varepsilon}(f) \leqslant \varrho_{\#, U}^{\varepsilon}\left((1+\delta) g_{\nu}\right)+c_{\delta} \varrho_{\#, U}^{\varepsilon}\left(f-g_{\nu}\right)
$$

for all $\delta>0$, where we also used the monotonicity of $\varphi(x, \cdot)$. Invoking now Lemmas 3.1 and 3.3 , we conclude that

$\limsup _{\varepsilon \rightarrow 0^{+}} \varrho_{\#, U}^{\varepsilon}(f) \leqslant \varrho_{\varphi(\cdot), U}\left(c_{n}(1+\delta)\left|\nabla g_{\nu}\right|\right)+c_{\delta} \max \left\{\left\|\nabla\left(f-g_{\nu}\right)\right\|_{\varphi(\cdot), U}^{\varphi_{\uparrow}},\left\|\nabla\left(f-g_{\nu}\right)\right\|_{\varphi(\cdot), U}^{\varphi_{\downarrow}}\right\}$.

Because $\left\|\nabla\left(f-g_{\nu}\right)\right\|_{\varphi(\cdot), U} \rightarrow 0$, it follows from (aDec) that $\lim _{\nu \rightarrow \infty} \varrho_{\varphi(\cdot), U}\left(c_{n}(1+\delta) \mid \nabla(f-\right.$ $\left.g_{\nu} \mid\right)=0$. Thus, letting $\nu \rightarrow \infty$ in (4.2) and using (2.5) again, we obtain

$$
\limsup _{\varepsilon \rightarrow 0^{+}} \varrho_{\#, U}^{\varepsilon}(f) \leqslant \varrho_{\varphi(\cdot), U}\left(c_{n}(1+\delta)^{2}|\nabla f|\right) \leqslant \varrho_{\varphi(\cdot), \Omega}\left(c_{n}(1+\delta)^{2}|\nabla f|\right) .
$$

By (aDec), $\varphi\left(x, c_{n}(1+\delta)^{2}|\nabla f(x)|\right) \leqslant c \varphi\left(x, c_{n}|\nabla f(x)|\right)$. This proves that $\lim \sup _{\varepsilon \rightarrow 0^{+}} \varrho_{\#, U}^{\varepsilon}(f) \leqslant$ $c \varrho_{\varphi(\cdot), U}\left(c_{n}|\nabla f|\right)$ for a general $\varphi$. If, in addition, $\varphi(x, \cdot)$ is continuous, we use $c \varphi\left(x, c_{n}|\nabla f(x)|\right)$ as a majorant and let $\delta \rightarrow 0$ in (4.3). Then, Lebesgue's dominated convergence theorem yields

$$
\limsup _{\varepsilon \rightarrow 0^{+}} \varrho_{\#, U}^{\varepsilon}(f) \leqslant \varrho_{\varphi(\cdot), \Omega}\left(c_{n}|\nabla f|\right) .
$$

We claim that

$$
\limsup _{U \nearrow \Omega} \limsup _{\varepsilon \rightarrow 0^{+}} \int_{0}^{\infty} \int_{\Omega_{r} \backslash U_{r}} \varphi\left(x, \frac{1}{r} M_{B(x, r)}^{\#} f\right) d x \psi_{\varepsilon}(r) d r=0,
$$

which, together with the bound on $\limsup _{\varepsilon \rightarrow 0^{+}} \varrho_{\#, U}^{\varepsilon}(f)$ established above, concludes the proof of the upper bound.

To prove (4.4), we observe that replacing $f$ by $\tilde{f}:=\|\nabla f\|_{\varphi(\cdot), \Omega}^{-1} f$ if necessary, we may assume that $\|\nabla f\|_{\varphi(\cdot), \Omega} \leqslant 1$. Indeed, by (aDec), if (4.4) holds for $\tilde{f}$, then it also holds for $f$. Arguing as in Lemma 3.3, we find that

$$
\begin{aligned}
& \int_{0}^{\infty} \int_{\Omega_{r} \backslash U_{r}} \varphi\left(x, \frac{1}{r} M_{B(x, r)}^{\#} f\right) d x \psi_{\varepsilon}(r) d r \\
& \quad \leqslant c \int_{0}^{\infty} \int_{\Omega_{r} \backslash U_{r}}\left(f_{B(x, r)} \varphi(y,|\nabla f(y)|) d y+h(x)+f_{B(x, r)} h(y) d y\right) d x \psi_{\varepsilon}(r) d r .
\end{aligned}
$$


Let $\gamma>0$. If $r \leqslant \frac{\gamma}{2}$, then $y \in \Omega \backslash U_{\gamma}$ whenever $x \in \Omega_{r} \backslash U_{r}$. Therefore, we may estimate the right-hand side of (4.5) as follows:

$$
\begin{aligned}
\int_{0}^{\infty} & \int_{\Omega_{r} \backslash U_{r}}\left(f_{B(x, r)} \varphi(y,|\nabla f(y)|) d y+h(x)+f_{B(x, r)} h(y) d y\right) d x \psi_{\varepsilon}(r) d r \\
\leqslant & \int_{0}^{\infty} \int_{\Omega_{r} \backslash U_{r}}\left(\int_{\Omega \backslash U_{\gamma}}(\varphi(y,|\nabla f(y)|)+h(y)) \frac{\chi_{B(y, r)}(x)}{|B(0, r)|} d y+h(x)\right) d x \psi_{\varepsilon}(r) d r \\
& +\int_{\frac{\gamma}{2}}^{\infty} \int_{\Omega_{r}}\left(f_{B(x, r)} \varphi(y,|\nabla f(y)|) d y+h(x)+f_{B(x, r)} h(y) d y\right) d x \psi_{\varepsilon}(r) d r
\end{aligned}
$$

Changing the order of integration as in Lemma 3.3, from (4.5) and (4.6), we obtain

$$
\begin{aligned}
& \int_{0}^{\infty} \int_{\Omega_{r} \backslash U_{r}} \varphi\left(x, \frac{1}{r} M_{B(x, r)}^{\#} f\right) d x \psi_{\varepsilon}(r) d r \\
& \quad \lesssim \varrho_{\varphi(\cdot), \Omega \backslash U_{\gamma}}(|\nabla f|)+2\|h\|_{L^{1}\left(\Omega \backslash U_{\gamma}\right)}+\left(\varrho_{\varphi(\cdot), \Omega}(|\nabla f|)+2\|h\|_{L^{1}(\Omega)}\right) \int_{\frac{\gamma}{2}}^{\infty} \psi_{\varepsilon}(r) d r .
\end{aligned}
$$

Then, (4.4) follows by (1.2) as $\varepsilon \rightarrow 0, \gamma \rightarrow 0$, and $U \rightarrow \Omega$, in this order.

It remains to prove the lower bound. The proof of this estimate is similar to the proof of the upper bound once we show that for all $U \subset \subset \Omega$, we have

$$
\varrho_{\#, U}^{\varepsilon}(f) \geqslant \varrho_{\#, U}^{\varepsilon}\left(\frac{1}{(1+\delta)} g_{\nu}\right)-c_{\delta} \varrho_{\#, U}^{\varepsilon}\left(f-g_{\nu}\right),
$$

where $c_{\delta}=\frac{1}{c_{\downarrow}}\left(1+\frac{1}{\delta}\right)^{\varphi_{\downarrow}}$. Fix $r>0$ and $x \in U_{r}$, and set $a^{\prime}:=\frac{1}{r} M_{B(x, r)}^{\#} g_{\nu}$ and $b^{\prime}:=$ $\frac{1}{r} M_{B(x, r)}^{\#}\left(f-g_{\nu}\right)$. Note that $\frac{1}{r} M_{B(x, r)}^{\#} f \geqslant\left|a^{\prime}-b^{\prime}\right|$. We consider two cases:

- If $a^{\prime}-b^{\prime} \geqslant 0$, then using (2.5) with $a=\frac{a^{\prime}-b^{\prime}}{1+\delta}$ and $b=\frac{b^{\prime}}{1+\delta}$, we obtain

$$
\varphi\left(x, a^{\prime}-b^{\prime}\right) \geqslant \varphi\left(x, \frac{a^{\prime}}{1+\delta}\right)-c_{\delta} \varphi\left(x, \frac{b^{\prime}}{1+\delta}\right) \geqslant \varphi\left(x, \frac{a^{\prime}}{1+\delta}\right)-c_{\delta} \varphi\left(x, b^{\prime}\right),
$$

where in the last inequality we used the fact that $\varphi(x, \cdot)$ is increasing and $\frac{1}{1+\delta} \leqslant 1$.

- If $a^{\prime}-b^{\prime} \leqslant 0$, then

$$
\varphi\left(x, \frac{a^{\prime}}{1+\delta}\right) \leqslant \varphi\left(x, \frac{b^{\prime}}{1+\delta}\right) \leqslant c_{\delta} \varphi\left(x, b^{\prime}\right)
$$

because $\varphi(x, \cdot)$ is increasing, $\frac{1}{1+\delta} \leqslant 1$, and $c_{\delta} \geqslant 1$. Thus,

$$
0 \geqslant \varphi\left(x, \frac{a^{\prime}}{1+\delta}\right)-c_{\delta} \varphi\left(x, b^{\prime}\right)
$$

Splitting the inner integral defining $\varrho_{\#, U}^{\varepsilon}(f)$ according to these two cases, we conclude that (4.7) holds.

Remark 4.2. In the proof of the previous proposition the assumption (aInc) was used only for the density of smooth functions in the Sobolev space. Presumably, (aInc) is not really needed for this, but it was used in the cited reference.

Proposition 4.3. Under the assumptions of Proposition 4.1, we have

$$
\lim _{\varepsilon \rightarrow 0^{+}}\|f\|_{\#, \Omega}^{\varepsilon} \approx c_{n}\|\nabla f\|_{\varphi(\cdot), \Omega} .
$$

If, in addition, $\varphi \in \Phi(\Omega)$, then (4.8) holds with equality.

Proof. Suppose first that $\varphi \in \Phi(\Omega)$. Since $\varphi$ is also doubling, $\varphi(x, \cdot)$ is continuous; thus, the modular inequality (4.1) holds with equality. Fixing $\delta>0$ and applying this equality to the function

$$
g_{\delta}:=\frac{f}{c_{n}(1+\delta)\left(\|\nabla f\|_{\varphi(\cdot), \Omega}+\delta\right)},
$$


we obtain

$$
\lim _{\varepsilon \rightarrow 0^{+}} \varrho_{\#, \Omega}^{\varepsilon}\left(g_{\delta}\right)=\varrho_{\varphi(\cdot), \Omega}\left(c_{n}\left|\nabla g_{\delta}\right|\right) \leqslant \frac{1}{1+\delta} \int_{\Omega} \varphi\left(x, \frac{|\nabla f|}{\|\nabla f\|_{\varphi(\cdot), \Omega}+\delta}\right) d x<1
$$

where we used convexity in the last estimate. Thus, $\varrho_{\#, \Omega}^{\varepsilon}\left(g_{\delta}\right) \leqslant 1$ for all sufficiently small $\varepsilon>0$. Consequently, also $\left\|g_{\delta}\right\|_{\#, \Omega}^{\varepsilon_{n}} \leqslant 1$ for all sufficiently small $\varepsilon>0$; that is, $\|f\|_{\#, \Omega}^{\varepsilon_{n}} \leqslant c_{n}(1+\delta)\left(\|\nabla f\|_{\varphi(\cdot), \Omega}+\delta\right)$. Letting $\varepsilon \rightarrow 0$ first and then $\delta \rightarrow 0$, we obtain $\lim \sup _{\varepsilon \rightarrow 0^{+}}\|f\|_{\#, \Omega}^{\varepsilon} \leqslant c_{n}\|\nabla f\|_{\varphi(\cdot), \Omega}$.

Next, we prove the opposite inequality. Fix $\delta>0$, let $\varepsilon_{j} \rightarrow 0$ as $j \rightarrow \infty$ be such that $\liminf \sin _{\varepsilon \rightarrow 0^{+}}\|f\|_{\#, \Omega}^{\varepsilon}=\lim _{j \rightarrow \infty}\|f\|_{\#, \Omega}^{\varepsilon_{j}}$, and set

$$
g_{\delta}:=\frac{f}{\lim _{j \rightarrow \infty}\|f\|_{\#, \Omega}^{\varepsilon_{j}}+\delta} .
$$

Because $\lim _{j \rightarrow \infty}\left\|g_{\delta}\right\|_{\#, \Omega}^{\varepsilon_{j}}<1$, we conclude that $\left\|g_{\delta}\right\|_{\#, \Omega}^{\varepsilon_{j}} \leqslant 1$ for all sufficiently large $j \in \mathbb{N}$. For all such $j \in \mathbb{N}, \varrho_{\#, \Omega}^{\varepsilon_{j}}\left(g_{\delta}\right) \leqslant 1$ by the definition of the norm. Letting $j \rightarrow \infty$, the previous proposition yields $\varrho_{\varphi(\cdot), \Omega}\left(c_{n}\left|\nabla g_{\delta}\right|\right) \leqslant 1$; so, $\left\|c_{n}\left|\nabla g_{\delta}\right|\right\|_{\varphi(\cdot), \Omega} \leqslant 1$. Therefore, $\left\|c_{n}|\nabla f|\right\|_{\varphi(\cdot), \Omega} \leqslant \lim _{j \rightarrow \infty}\|f\|_{\#, \Omega}^{\varepsilon_{j}}+\delta=\liminf _{\varepsilon \rightarrow 0^{+}}\|f\|_{\#, \Omega}^{\varepsilon}+\delta$. Letting $\delta \rightarrow 0$, we obtain the desired inequality.

This completes the proof in the case $\varphi \in \Phi(\Omega)$. If $\varphi \in \Phi_{w}(\Omega)$, we find $\psi \in \Phi(\Omega)$ with $\varphi \simeq \psi\left(\right.$ Remarks 2.3 and 2.7). Then, $\|\nabla f\|_{\varphi(\cdot)} \approx\|\nabla f\|_{\psi(\cdot)}$ and similarly for the \#-norm; so, the claim follows from the first part.

The next lemma shows that the condition $\lim \sup _{\varepsilon \rightarrow 0^{+}} \varrho_{\#, \Omega}^{\varepsilon}(f)<\infty$ implies that $f$ is locally in a Sobolev space. The estimate for the norm obtained in this way is not uniform, however. Nevertheless, this information is used later to prove a uniform estimate.

Lemma 4.4. Let $\Omega \subset \mathbb{R}^{n}$ be open, let $\varphi \in \Phi_{w}(\Omega)$ satisfy Assumptions (A), (aInc), and (aDec), and let $\left(\psi_{\varepsilon}\right)_{\varepsilon}$ be a family of functions satisfying (1.1) and (1.2). Assume that $f \in L_{\mathrm{loc}}^{1}(\Omega)$ and

$$
\limsup _{\varepsilon \rightarrow 0^{+}} \varrho_{\#, \Omega}^{\varepsilon}(f)<\infty
$$

Then, there is a constant $c>0$ such that, for every $U \subset \subset \Omega, \int_{U} \varphi_{U}^{-}(|\nabla f(x)|) d x \leqslant c$. Moreover, $\nabla f \in L_{\mathrm{loc}}^{\varphi_{\uparrow}}\left(\Omega ; \mathbb{R}^{n}\right)$.

Proof. Let $U \subset \subset \Omega$ and note that $\varphi_{U}^{-} \in \Phi_{w}$ (the only nontrivial condition is the limit at infinity, which follows from (A0)). By [19, Lemma 2.2], there exists $\xi \in \Phi$ with $\xi \simeq \varphi_{U}^{-}$for which the equivalent constant depends only on the monotonicity constant of $t \mapsto \frac{\varphi(x, t)}{t}$. Then, by (aDec), we can find $c>0$, independent of $U$, such that $c^{-1} \varphi_{U}^{-} \leqslant \xi \leqslant c \varphi_{U}^{-}$.

Fix $\delta>0$, and let $G_{\delta}$ be a standard mollifier; that is, $G_{\delta}(x)=\delta^{-n} G(x / \delta)$, where $G \in C_{0}^{\infty}(B(0,1))$ is a non-negative function, radially symmetric, and $\int_{B(0,1)} G(x) d x=1$. Let $\tau>0$ be such that $U \subset \subset \Omega_{\tau}$. Then, for $0<\delta<\tau, G_{\delta} * f \in C^{\infty}\left(\Omega_{\tau}\right)$ and, by Lemma 3.1 with $\varphi=\xi, U:=U_{\delta}$, and $\Omega:=\Omega_{\tau}$,

$$
\begin{aligned}
\lim _{\varepsilon \rightarrow 0^{+}} \int_{0}^{\infty} \int_{U_{\delta+r}} \xi\left(\frac{1}{r} M_{B(x, r)}^{\#}\left(G_{\delta} * f\right)\right) d x \psi_{\varepsilon}(r) d r & =\varrho_{\xi, U_{\delta}}\left(c_{n}\left|\nabla\left(G_{\delta} * f\right)\right|\right) \\
& \geqslant \varrho_{\xi, U_{\tau}}\left(c_{n}\left|\nabla\left(G_{\delta} * f\right)\right|\right)
\end{aligned}
$$


On the other hand, by the triangle inequality and a change on the order of integration, we obtain, for all $r>0$ and $x \in U_{r}$,

$$
\begin{aligned}
M_{B(x, r)}^{\#}\left(G_{\delta} * f\right) & =f_{B(x, r)}\left|\left(G_{\delta} * f\right)(y)-\left(G_{\delta} * f\right)_{B(x, r)}\right| d y \\
& =f_{B(x, r)}\left|\left(G_{\delta} * f\right)(y)-\left(G_{\delta} * f_{B(\cdot, r)}\right)(x)\right| d y \leqslant\left(G_{\delta} * M_{B(\cdot, r)}^{\#} f\right)(x)
\end{aligned}
$$

Hence, the monotonicity of $\xi$ yields

$$
\xi\left(\frac{1}{r} M_{B(x, r)}^{\#}\left(G_{\delta} * f\right)\right) \leqslant \xi\left(\left(G_{\delta} * \frac{1}{r} M_{B(\cdot, r)}^{\#} f\right)(x)\right) .
$$

For $x \in \Omega_{r}$, define $g_{r}(x):=\frac{1}{r} M_{B(x, r)}^{\#} f$. Since $G_{\delta} d x$ is a probability measure and $\xi$ is convex,

$$
\xi\left(\frac{1}{r} M_{B(x, r)}^{\#}\left(G_{\delta} * f\right)\right) \leqslant \xi\left(\left(G_{\delta} * g_{r}\right)(x)\right) \leqslant\left(G_{\delta} * \xi\left(g_{r}\right)\right)(x) .
$$

Integrating $G_{\delta} * \xi\left(g_{r}\right)$ over $U_{r+\delta}$ and changing variables and the order of integration, we obtain

$$
\begin{aligned}
\int_{U_{\delta+r}} \int_{B(x, \delta)} G_{\delta}(x-z) \xi\left(g_{r}(z)\right) d z d x & =\int_{B(0, \delta)} G_{\delta}(w) \int_{U_{\delta+r}} \xi\left(g_{r}(x-w)\right) d x d w \\
& \leqslant \int_{U_{r}} \xi\left(g_{r}(y)\right) d y .
\end{aligned}
$$

Combining the previous two estimates, we conclude that

$$
\int_{U_{\delta+r}} \xi\left(\frac{1}{r} M_{B(x, r)}^{\#}\left(G_{\delta} * f\right)\right) d x \leqslant \int_{U_{r}} \xi\left(g_{r}(x)\right) d x \leqslant c \int_{U_{r}} \varphi\left(x, \frac{1}{r} M_{B(x, r)}^{\#} f\right) d x
$$

where in the last inequality we used the definition of $g_{r}$ and the estimate $\xi(t) \leqslant c \varphi(x, t)$ for $x \in U$. Consequently,

$$
\limsup _{\varepsilon \rightarrow 0^{+}} \int_{0}^{\infty} \int_{U_{\delta+r}} \xi\left(\frac{1}{r} M_{B(x, r)}^{\#}\left(G_{\delta} * f\right)\right) d x \psi_{\varepsilon}(r) d r \leqslant c \limsup _{\varepsilon \rightarrow 0^{+}} \varrho_{\#, U}^{\varepsilon}(f) \leqslant c \limsup _{\varepsilon \rightarrow 0^{+}} \varrho_{\#, \Omega}^{\varepsilon}(f)<\infty,
$$

which, together with (4.9), shows that $\left(\nabla\left(G_{\delta} * f\right)\right)_{\delta}$ is bounded in $L^{\xi}\left(U_{\tau} ; \mathbb{R}^{n}\right)$.

By (A0), (aInc), and (aDec), $L^{\xi}\left(U_{\tau} ; \mathbb{R}^{n}\right)$ is reflexive (see [19, Lemma 2.4 and Proposition 4.6]). Since $\left(\nabla\left(G_{\delta} * f\right)\right)_{\delta}$ is a bounded sequence, it has a subsequence which converges weakly in $L^{\xi}\left(U_{\tau} ; \mathbb{R}^{n}\right)$ to a function $l \in L^{\xi}\left(U_{\tau} ; \mathbb{R}^{n}\right)$. Using the fact that $G_{\delta} * f$ converges to $f$ in $L^{1}(U)$, it follows from the definition of weak derivative that $l=\nabla f$. By weak semi-continuity [14, Theorem 2.2.8], (4.9), and (4.11), we obtain that

$$
\begin{aligned}
\int_{U_{\tau}} \varphi_{U}^{-}\left(c_{n}|\nabla f(x)|\right) d x & \leqslant c \rho_{\xi, U_{\tau}}\left(c_{n}|\nabla f|\right) \leqslant c \liminf _{\delta \rightarrow 0^{+}} \rho_{\xi, U_{\tau}}\left(c_{n}\left|\nabla\left(G_{\delta} * f\right)\right|\right) \\
& \leqslant c \limsup _{\varepsilon \rightarrow 0^{+}} \varrho_{\#, \Omega}^{\varepsilon}(f)<\infty .
\end{aligned}
$$

Letting $\tau \rightarrow 0$, we obtain $\int_{U} \varphi_{U}^{-}(|\nabla f(x)|) d x \leqslant c$ by monotone convergence and (aDec).

Finally, we observe that Assumptions (aInc) and (A0) imply that $t^{\varphi \uparrow} \leqslant c\left(\varphi_{U}^{-}(t)+1\right)$ with $c>0$ independent of $U$. Hence,

$$
\int_{U}|\nabla f(x)|^{\varphi_{\uparrow}} d x \leqslant c \int_{U} \varphi_{U}^{-}(|\nabla f(x)|)+1 d x \leqslant c+c|U|
$$

which yields $\nabla f \in L_{\mathrm{loc}}^{\varphi_{\uparrow}}\left(\Omega ; \mathbb{R}^{n}\right)$.

We now remove the local-condition from the previous lemma: 
Proposition 4.5. Let $\Omega \subset \mathbb{R}^{n}$ be open, let $\varphi \in \Phi_{w}(\Omega)$ satisfy Assumptions (A), (aInc), and (aDec), and let $\left(\psi_{\varepsilon}\right)_{\varepsilon}$ be a family of functions satisfying (1.1) and (1.2). Assume that $f \in L_{\mathrm{loc}}^{1}(\Omega)$ and

$$
\limsup _{\varepsilon \rightarrow 0^{+}} \varrho_{\#, \Omega}^{\varepsilon}(f)<\infty
$$

Then, $\nabla f \in L^{\varphi(\cdot)}\left(\Omega ; \mathbb{R}^{n}\right)$.

Proof. Let $U \subset \subset V \subset \subset \Omega$ and $\delta \in\left(0, \frac{1}{6}\right)$ be such that $U^{\delta} \subset V \subset \Omega_{\delta}$. Let $G_{\delta}$ and $g_{r}$ be as in the previous lemma. By Remarks 2.3 and 2.7, there exists $\xi \in \Phi(\Omega)$ equivalent to $\varphi$ which satisfies the same assumptions. In Lemma 4.4, we proved that $\int_{U} \xi_{U}^{-}(|\nabla f|) d x \leqslant c$ for every $U \subset \subset \Omega$. In view of (aDec), by scaling the function $f$, if necessary, we may assume that

$$
\int_{B} \xi_{B}^{-}(|\nabla f(x)|) d x \leqslant 3^{-n} \text { for every ball } B \subset \subset \Omega \text { with }|B| \leqslant 1 .
$$

Let $r \in(0, \delta]$ and $x \in U_{r}$. By the Poincaré and triangle inequalities,

$$
\frac{1}{r} M_{B(x, r)}^{\#}\left(G_{\delta} * f\right) \leqslant c f_{B(x, r)}\left|\nabla\left(G_{\delta} * f\right)(y)\right| d y \leqslant c f_{B(x, r)} \int_{B(y, \delta)} G_{\delta}(y-z)|\nabla f(z)| d z d y .
$$

Note that $G_{\delta} \leqslant c \frac{\chi_{B(0, \delta)}}{|B(0, \delta)|}$ hence, by [22, Lemma 4.3] and (4.12), together with the previous estimate, we obtain

$$
\begin{aligned}
\frac{1}{r} M_{B(x, r)}^{\#}\left(G_{\delta} * f\right) & \leqslant c f_{B(x, r)} f_{B(y, \delta)}|\nabla f(z)| d z d y \\
& \leqslant c f_{B(x, r)}\left(\xi_{B(y, \delta)}^{-}\right)^{-1}\left(f_{B(y, \delta)} \xi_{B(y, \delta)}^{-}(|\nabla f(z)|) d z\right) d y \\
& \leqslant c f_{B(x, r)}\left(\xi_{B(y, \delta)}^{-}\right)^{-1}\left(\frac{1}{3^{n}|B(y, \delta)|}\right) d y
\end{aligned}
$$

Observe that $B(y, \delta) \subset B(x, 3 \delta)$ for every $y \in B(x, r)$, and $|B(y, \delta)|=|B(x, \delta)|$ and $|B(x, 3 \delta)|=3^{n}|B(x, \delta)|$. By Lemmas 2.9 and 2.10,

$$
\left(\xi_{B(y, \delta)}^{-}\right)^{-1}\left(\frac{1}{3^{n}|B(y, \delta)|}\right) \approx\left(\xi_{B(x, 3 \delta)}^{-}\right)^{-1}\left(\frac{1}{3^{n}|B(x, \delta)|}\right) \approx\left(\xi_{B(x, \delta)}^{-}\right)^{-1}\left(\frac{1}{3^{n}|B(x, \delta)|}\right)
$$

when $y \in B(x, r)$. Then, because $\left(\xi_{B(x, \delta)}^{-}\right)^{-1}$ is increasing, we obtain

$$
\frac{1}{r} M_{B(x, r)}^{\#}\left(G_{\delta} * f\right) \leqslant c\left(\xi_{B(x, \delta)}^{-}\right)^{-1}\left(3^{-n}|B(x, \delta)|^{-1}\right) \leqslant c\left(\xi_{B(x, \delta)}^{-}\right)^{-1}\left(|B(x, \delta)|^{-1}\right) .
$$
Thus,

On the other hand, by (4.10), we have also $\frac{1}{r} M_{B(x, r)}^{\#}\left(G_{\delta} * f\right) \leqslant\left(G_{\delta} * g_{r}\right)(x) \leqslant c f_{B(x, \delta)}\left|g_{r}\right| d y$.

$$
\frac{1}{r} M_{B(x, r)}^{\#}\left(G_{\delta} * f\right) \leqslant c \min \left\{f_{B(x, \delta)}\left|g_{r}\right| d y,\left(\xi_{B(x, \delta)}^{-}\right)^{-1}\left(\frac{1}{|B(x, \delta)|}\right)\right\} .
$$

Then, by the monotonicity of $\xi(x, \cdot)$ and Lemma 2.12, invoking (aDec) if necessary, it follows that

$$
\xi\left(x, \frac{1}{r} M_{B(x, r)}^{\#}\left(G_{\delta} * f\right)\right) \leqslant c\left(f_{\substack{B(x, \delta) \\ 15}}\left(\xi\left(y, g_{r}(y)\right)+h(y)\right) d y+h(x)\right)
$$


Integrating this estimate over $x \in U_{r}$, changing the order of integration as in Lemma 3.3, and using the inclusion $\left(U_{r}\right)^{\delta} \subset V_{r}$, we obtain

$$
\begin{aligned}
\int_{U_{r}} \xi\left(x, \frac{1}{r} M_{B(x, r)}^{\#}\left(G_{\delta} * f\right)\right) d x & \leqslant c\left(\int_{\left(U_{r}\right)^{\delta}} \xi\left(y, g_{r}(y)\right)+h(y) d y+\int_{U_{r}} h(x) d x\right) \\
& \leqslant c\left(\int_{V_{r}} \xi\left(y, \frac{1}{r} M_{B(y, r)}^{\#} f\right) d y+2\|h\|_{L^{1}(\Omega)}\right)
\end{aligned}
$$

for $r \leqslant \delta$.

Next, we consider $r \in(\delta, \infty)$. Fix $x \in U_{r}$, and recall that $\nabla f \in L^{\varphi_{\uparrow}}\left(V ; \mathbb{R}^{n}\right)$ by Lemma 4.4. By the Poincaré and Hölder inequalities, we have

$$
\begin{aligned}
\frac{1}{r} M_{B(x, r)}^{\#}\left(G_{\delta} * f\right) & \leqslant c f_{B(x, r)}\left|\nabla\left(G_{\delta} * f\right)(y)\right| d y \leqslant c f_{B(x, r)}\left\|G_{\delta}\right\|_{\left(\varphi_{\uparrow}\right)^{\prime}, B(0, \delta)}\|\nabla f\|_{\varphi_{\uparrow}, B(y, \delta)} d y \\
& \leqslant c_{\delta}\|\nabla f\|_{\varphi_{\uparrow}, U^{\delta}} \leqslant c_{\delta}\|\nabla f\|_{\varphi_{\uparrow}, V} .
\end{aligned}
$$

Hence,

for $r>\delta$.

$$
\int_{U_{r}} \xi\left(x, \frac{1}{r} M_{B(x, r)}^{\#}\left(G_{\delta} * f\right)\right) d x \leqslant \int_{U_{r}} \xi\left(x, c_{\delta}\|\nabla f\|_{\varphi_{\uparrow}, V}\right) d x
$$

Combining the two cases, $r \leqslant \delta$ and $r>\delta$, we obtain

$$
\begin{aligned}
& \int_{0}^{\infty} \int_{U_{r}} \xi\left(x, \frac{1}{r} M_{B(x, r)}^{\#}\left(G_{\delta} * f\right)\right) d x \psi_{\varepsilon}(r) d r \\
& \quad \leqslant c \int_{0}^{\delta}\left(\int_{V_{r}} \xi\left(x, \frac{1}{r} M_{B(x, r)}^{\#} f\right) d x+2\|h\|_{L^{1}(\Omega)}\right) \psi_{\varepsilon}(r) d r+\int_{\delta}^{\infty} \int_{U_{r}} \xi\left(x, c_{\delta}\|\nabla f\|_{\varphi_{\uparrow}, V}\right) d x \psi_{\varepsilon}(r) d r .
\end{aligned}
$$

The second term on the right-hand side of the previous inequality tends to zero as $\varepsilon \rightarrow 0$ by (loc) and (1.2). Consequently,

$$
\limsup _{\varepsilon \rightarrow 0} \int_{0}^{\infty} \int_{U_{r}} \xi\left(x, \frac{1}{r} M_{B(x, r)}^{\#}\left(G_{\delta} * f\right)\right) d x \psi_{\varepsilon}(r) d r \leqslant c \limsup _{\varepsilon \rightarrow 0} \rho_{\#, V}^{\varepsilon}(f)+c<\infty
$$

by hypothesis, where we also used (1.1) and the fact that $\xi \approx \varphi$.

Thus, arguing as in Lemma 4.4, we conclude that $\nabla f \in L^{\xi(\cdot)}\left(U ; \mathbb{R}^{n}\right)$. Hence, also $\nabla f \in L^{\varphi(\cdot)}\left(U ; \mathbb{R}^{n}\right)$ (see Remark 2.6).

Finally, we appeal to Proposition 4.1 to conclude that

$$
\varrho_{\varphi(\cdot), U}\left(c_{n}|\nabla f|\right) \leqslant c \limsup _{\varepsilon \rightarrow 0^{+}} \varrho_{\#, U}^{\varepsilon}(f) \leqslant c \limsup _{\varepsilon \rightarrow 0^{+}} \varrho_{\#, \Omega}^{\varepsilon}(f)<\infty .
$$

Because the upper bound in the last estimate is independent of $U$, we conclude the proof of Proposition 4.5 by monotone convergence as $U \nearrow \Omega$.

Combining the propositions of this section, we arrive at the following theorem.

Theorem 4.6. Let $\Omega \subset \mathbb{R}^{n}$ be an open set, let $\varphi \in \Phi_{w}(\Omega)$ satisfy Assumptions (A), (aInc), and (aDec), and let $\left(\psi_{\varepsilon}\right)_{\varepsilon}$ be a family of functions satisfying (1.1) and (1.2). Assume that $f \in L_{\mathrm{loc}}^{1}(\Omega)$. Then,

$$
\nabla f \in L^{\varphi(\cdot)}\left(\Omega ; \mathbb{R}^{n}\right) \quad \Leftrightarrow \quad \limsup _{\varepsilon \rightarrow 0^{+}} \varrho_{\#, \Omega}^{\varepsilon}(f)<\infty .
$$

In this case,

$$
\lim _{\varepsilon \rightarrow 0^{+}} \varrho_{\#, \Omega}^{\varepsilon}(f) \approx \varrho_{\varphi(\cdot), \Omega}\left(c_{n}|\nabla f|\right) \quad \text { and } \quad \lim _{\varepsilon \rightarrow 0^{+}}\|f\|_{\#, \Omega}^{\varepsilon} \approx c_{n}\|\nabla f\|_{\varphi(\cdot), \Omega},
$$

where $c_{n}:=f_{B(0,1)}\left|x \cdot e_{1}\right| d x$. 


\section{ACKNOWLEDGEMENTS}

This work was partially supported by the Fundação para a Ciência e a Tecnologia (Portuguese Foundation for Science and Technology) through the projects UID/MAT/00297/ 2013 (Centro de Matemática e Aplicações) and EXPL/MAT-CAL/0840/2013. Part of this work was done while the authors enjoyed the hospitality of University of Turku and of the Centro de Matemática e Aplicações (CMA), Faculdade de Ciências e Tecnologia, Universidade Nova de Lisboa.

\section{REFERENCES}

[1] A. Almeida and P. Hästö: Besov spaces with variable smoothness and integrability, J. Funct. Anal. 258 (2010), no. 5, 1628-1655.

[2] G. Aubert and P. Kornprobst: Can the nonlocal characterization of Sobolev spaces by Bourgain et al. be useful for solving variational problems? SIAM J. Numer. Anal. 47, (2009), 844-860.

[3] P. Baroni, M. Colombo and G. Mingione: Harnack inequalities for double phase functionals. Nonlinear Anal. 121 (2015), 206-222.

[4] P. Baroni, M. Colombo and G. Mingione: Non-autonomous functionals, borderline cases and related function classes, St Petersburg Math. J. 27 (2016), 347-379.

[5] J. Bourgain, H. Brézis, and P. Mironescu: Another look at Sobolev spaces, Optimal control and partial differential equations. In honour of Professor Alain Bensoussan's 60th birthday. Proceedings of the conference, Paris, France, December 4, 2000 (2001), 439-455.

[6] J. Bourgain, H. Brézis, and P. Mironescu: Limiting embedding theorems for $W^{s, p}$ when $s \uparrow 1$ and applications, J. Anal. Math. 87 (2002), 77-101.

[7] Y. Chen, S. Levine, and R. Rao: Variable exponent, linear growth functionals in image restoration, SIAM J. Appl. Math. 66 (2006), no. 4, 1383-1406.

[8] M. Colombo and G. Mingione: Regularity for Double Phase Variational Problems Arch. Ration. Mech. Anal. 215 (2015), no. 2, 443-496.

[9] M. Colombo and G. Mingione: Bounded minimisers of double phase variational integrals, Arch. Ration. Mech. Anal. 218 (2015), no. 1, 219-273.

[10] M. Colombo and G. Mingione: Calderón-Zygmund estimates and non-uniformly elliptic operators, J. Funct. Anal. 270 (2016), 1416-1478.

[11] D. Cruz-Uribe and A. Fiorenza: Variable Lebesgue Spaces. Foundations and Harmonic Analysis, Birkhäuser/Springer, New York, 2013.

[12] D. Cruz-Uribe and P. Hästö: Extrapolation and interpolation in generalized Orlicz spaces, Preprint (2016).

[13] L. Diening and P. Hästö: Variable exponent trace spaces, Studia Math. 183 (2007) 127-141.

[14] L. Diening, P. Harjulehto, P. Hästö, and M. Růžičcka: Lebesgue and Sobolev Spaces with Variable Exponents, Lecture Notes in Mathematics, vol. 2017, Springer-Verlag, Berlin, 2011.

[15] L. Diening, P. Hästö and S. Roudenko: Function spaces of variable smoothness and integrability, J. Funct. Anal. 256 (2009), no. 6, 1731-1768.

[16] R. Ferreira, C. Kreisbeck and A.M. Ribeiro: Characterization of polynomials and higher-order Sobolev spaces in terms of functionals involving difference quotients, Nonlinear Analysis 112 (2015), 199-214.

[17] F. Giannetti and A. Passarelli di Napoli: Regularity results for a new class of functionals with non-standard growth conditions, J. Differential Equations 254 (2013) 1280-1305.

[18] P. Harjulehto and P. Hästö: Riesz potential in generalized Orlicz Spaces, Forum Math., to appear. DOI: 10.1515 /forum-2015-0239

[19] P. Harjulehto, P. Hästö and R. Klén: Generalized Orlicz spaces and related PDE, Nonlinear Anal. 143 (2016), 155-173.

[20] P. Harjulehto, P. Hästö, V. Latvala and O. Toivanen: Critical variable exponent functionals in image restoration, Appl. Math. Letters 26 (2013), 56-60.

[21] P. Harjulehto, P. Hästö and O. Toivanen: Hölder regularity of quasiminimizers under generalized growth conditions, Preprint (2016).

[22] P. Hästö: The maximal operator on generalized Orlicz spaces, J. Funct. Anal. 269 (2015), 4038-4048.

[23] P. Hästö: Corrigendum to "The maximal operator on generalized Orlicz spaces" [J. Funct. Anal. 269 (2015) 4038-4048], J. Funct. Anal. 271 (2016), no. 1, 240-243. 
[24] P. Hästö and A. Ribeiro: Characterization of the variable exponent Sobolev norm without derivatives, Commun. Contemp. Math., to appear. DOI: 10.1142/S021919971650022X

[25] G. Leoni and D. Spector: Characterization of Sobolev and BV spaces, J. Funct. Anal. 261 (10) (2011), 2926-2958.

[26] F.-Y. Maeda, Y. Mizuta, T. Ohno and T. Shimomura: Boundedness of maximal operators and Sobolev's inequality on Musielak-Orlicz-Morrey spaces, Bull. Sci. Math. 137 (2013), 76-96.

[27] Y. Mizuta, T. Ohno and T. Shimomura: Sobolev's inequalities and vanishing integrability for Riesz potentials of functions in the generalized Lebesgue space $L^{p(\cdot)} \log L^{q(\cdot)}$, J. Math. Anal. Appl. 345 (2008), 70-85.

[28] J. Musielak: Orlicz spaces and modular spaces, Lecture Notes in Mathematics, 1034. Springer, Berlin, 1983.

[29] J. Ok: Gradient estimates for elliptic equations with $L^{p(\cdot)} \log L$ growth, Calc. Var. Partial Differential Equations 55 (2016), no. 2, 1-30.

[30] J. Ok: Regularity results for a class of obstacle problems with nonstandard growth, J. Math. Anal. Appl. 444 (2016), no. 2, 957-979.

[31] L. Rudin, S. Osher, and E. Fatemi: Nonlinear total variation based noise removal algorithms, Physica D 60, (1992), no.1-4, 259-268.

[32] A. Świerczewska-Gwiazda: Nonlinear parabolic problems in Musielak-Orlicz spaces, Nonlinear Anal. 98 (2014), 48-65.

(R. Ferreira) King Abdullah University of Science and Technology (KAUST), CEMSE Division, Thuwal 23955-6900, SAUdi Arabia.

E-mail address: rita.ferreira@kaust.edu.sa

$U R L:$ http://www.ritaferreira.pt

(P. Hästö) Department of Mathematical Sciences, P.O. Box 3000, Fi-90014 University of Oulu, Finland, and, Department of Mathematics and Statistics, University of Turku, FINLAND

E-mail address: peter.hasto@oulu.fi

URL: http://cc.oulu.fi/ phasto/

(A.M. Ribeiro) Centro de Matemática e AplicaÇões (CMA) and Departamento de Matemática, Faculdade de Ciências e Tecnologia, Universidade Nova de lisboa, Quinta da Torre, 2829-516 Caparica, Portugal

E-mail address: amfr@fct.unl.pt

$U R L:$ https://sites.google.com/site/anaribeirowebpage/home 